\title{
Stellar Evolution at Low Metallicity
}

\author{
Raphael Hirschi ${ }^{1}$, Cristina Chiappini ${ }^{2,3}$, Georges Meynet ${ }^{2}$, \\ André Maeder ${ }^{2}$ and Sylvia Ekström ${ }^{2}$ \\ ${ }^{1}$ Astrophysics group, Keele University, Lennard-Jones Lab., Keele, ST5 5BG, UK \\ email: r.hirschi@epsam.keele.ac.uk \\ ${ }^{2}$ Observatoire Astronomique de l'Université de Genève, CH-1290, Sauverny, Switzerland \\ ${ }^{3}$ Osservatorio Astronomico di Trieste, Via G. B. Tiepolo 11, I - 34131 Trieste, Italia
}

\begin{abstract}
Massive stars played a key role in the early evolution of the Universe. They formed with the first halos and started the re-ionisation. It is therefore very important to understand their evolution. In this review, we first recall the effect of metallicity $(Z)$ on the evolution of massive stars. We then describe the strong impact of rotation induced mixing and mass loss at very low $Z$. The strong mixing leads to a significant production of primary ${ }^{14} \mathrm{~N},{ }^{13} \mathrm{C}$ and ${ }^{22} \mathrm{Ne}$. Mass loss during the red supergiant stage allows the production of Wolf-Rayet stars, type Ib,c supernovae and possibly gamma-ray bursts (GRBs) down to almost $Z=0$ for stars more massive than $60 \mathrm{M}_{\odot}$. Galactic chemical evolution models calculated with models of rotating stars better reproduce the early evolution of $\mathrm{N} / \mathrm{O}, \mathrm{C} / \mathrm{O}$ and ${ }^{12} \mathrm{C} /{ }^{13} \mathrm{C}$. Finally, the impact of magnetic fields is discussed in the context of GRBs.
\end{abstract}

Keywords. stars: mass loss - stars: Population II - stars: rotation - supernovae: general - stars: Wolf-Rayet - Galaxy: evolution - gamma rays: bursts

\section{Introduction}

Massive stars started forming about 400 millions years after the Big Bang and ended the dark ages by re-ionising the Universe. They therefore played a key role in the early evolution of the Universe and it is important to understand the properties and the evolution of the first stellar generations to determine the feedback they had on the formation of the first cosmic structures. It is unfortunately not possible to observe the first massive stars because they died a long time ago but their chemical signature can be observed in low mass halo stars (called EMP stars), which are so old and metal poor that the interstellar medium out of which these halo stars formed are thought to have been enriched by one or a few massive stars. Since the re-ionisation, massive stars have continuously injected kinetic energy (via various types of supernovae) and newly produced chemical elements (by both hydrostatic and explosive burning and $\mathrm{s}$ and $\mathrm{r}$ processes) into the interstellar medium of their host galaxy. They are thus important players for the chemodynamical evolution of galaxies. Most massive stars leave a remnant at their death, either a neutron star or a black hole, which produce pulsar or X-ray binaries.

The evolution of stars is governed by three main parameters, which are the initial mass, metallicity $(Z)$ and rotation rate. The evolution is also influenced by the presence of magnetic fields and of a close binary companion. For massive stars $\left(M \gtrsim 10 \mathrm{M}_{\odot}\right)$ around solar metallicity mass loss plays a crucial role, in some cases removing more than half of the initial mass. Internal mixing, induced mainly by convection and rotation also significantly affect the evolution of stars. In this review, after a summary of the properties of low $Z$ stars, we discuss the possible impact of rotation induced mixing and mass loss at low $Z$. We then present the implication of strong mixing and mass loss for the nucleosynthesis and for galactic chemical evolution in the context of extremely metal 
poor stars. We also discuss the effects of magnetic fields. We end with conclusions and an outlook.

\section{Properties of non-rotating low- $Z$ stars}
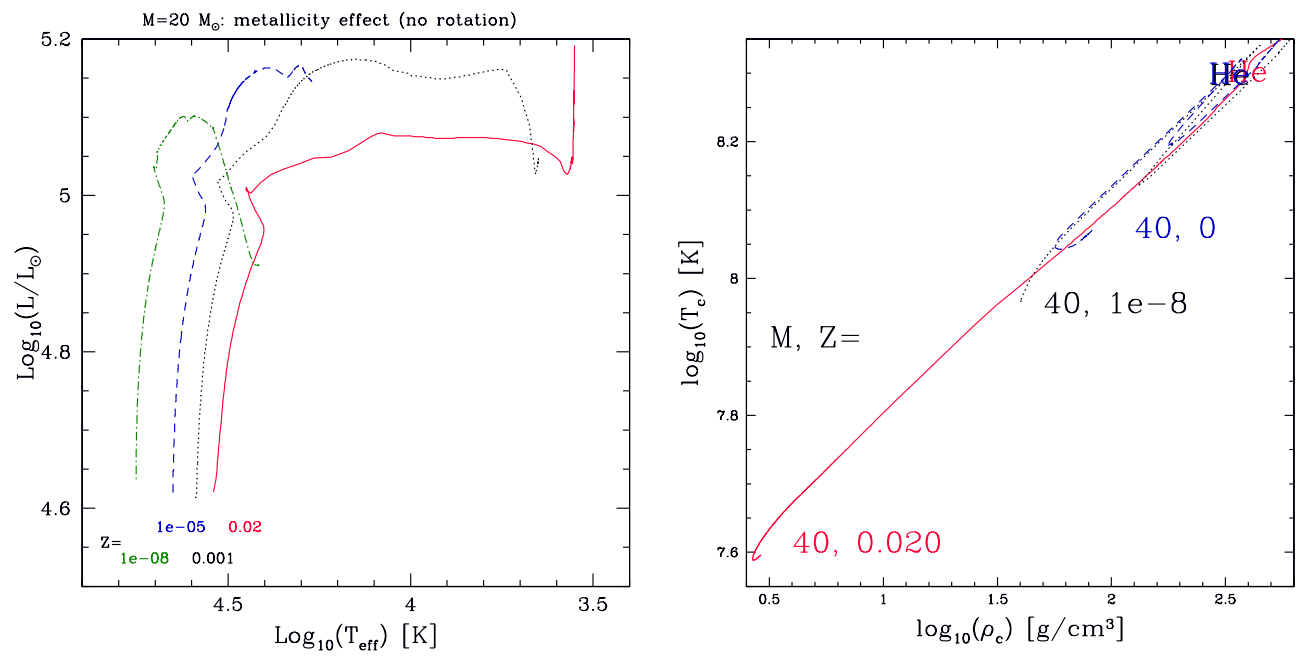

Figure 1. Left: H-R diagram for non-rotating $20 \mathrm{M}_{\odot}$ models with $Z=10^{-8}, 10^{-5}, 0.001$ and 0.02 , showing that more metal poor stars have more compact envelopes and are less likely to reach the red supergiant stage. Right: Central temperature versus central density diagram for 40 $\mathrm{M}_{\odot}$ models with $Z=0,10^{-8}$ and 0.02 . The evolutionary tracks start where hydrogen burning start and the He symbols are placed at the start of helium burning. The central conditions are much hotter and denser at very low $Z$. Note also the different initial H-burning conditions between $Z=0$ and $10^{-8}$ (explained in the text).

The first stellar generations are different from solar metallicity stars due to their low metal content or absence of it. First, low- $Z$ stars are more compact (see Fig. 1) due to lower opacity. Second, metal free stars burn hydrogen in a core, which is denser and hotter due to the lack of initial CNO elements (see start of dashed curve in Fig. 1 right). This implies that the transition between core hydrogen and helium burning is much shorter and smoother. Furthermore, hydrogen burns via the pp-chain in shell burning. These differences make the metal free stars different from the second or later generation stars (Ekström et al. 2008).

Third, mass loss is metallicity dependent and therefore is expected to become very weak at very low metallicity. The metallicity $(Z)$ dependence of mass loss rates is usually described using the formula:

$$
\dot{M}(Z)=\dot{M}\left(Z_{\odot}\right)\left(Z / Z_{\odot}\right)^{\alpha}
$$

The exponent $\alpha$ varies between 0.5-0.6 (Kudritzki \& Puls 2000, Kudritzki 2002) and 0.7-0.86 (Vink et al. 2001, Vink \& de Koter 2005) for O-type and WR stars respectively (See Mokiem et al. 2007 for a recent comparison between mass loss prescriptions and observed mass loss rates). Until very recently, most models use at best the total metal content present at the surface of the star to determine the mass loss rate. However, the surface chemical composition becomes very different from the solar mixture, due either to mass loss in the WR stage or by internal mixing (convection and rotation) after the 
main sequence. It is therefore important to know the contribution from each chemical species to opacity and mass loss.

Recent studies (Vink et al. 2000, Vink \& de Koter 2005) show that iron is the dominant element concerning radiation line-driven mass loss for O-type and WR stars. In the case of WR stars, there is however a plateau at low metallicity due to the contributions from light elements like carbon, nitrogen and oxygen (CNO). In between the hot and cool parts of the HR-diagram, mass loss is not well understood. Observations of the LBV stage indicate that several solar masses per year may be lost (Smith et al. 2003) and there is no indication of a metallicity dependence. In the red supergiant (RSG) stage, the rates generally used are still those of Nieuwenhuijzen \& de Jager (1990). More recent observations indicate that there is a very weak dependence of dust-driven mass loss on metallicity and that CNO elements and especially nucleation seed components like silicon and titanium are dominant (van Loon 2000, van Loon 2006, Ferrarotti \& Gail 2006). Van Loon et al. (2005) provide recent mass loss rate prescriptions in the RSG stage. In particular, the ratio of carbon to oxygen is important to determine which kind of molecules and dusts form. If the ratio of carbon to oxygen is larger than one, then carbon-rich dust would form, and more likely drive a wind since they are more opaque than oxygen-rich dust at low metallicity (Höfner \& Andersen 2007).

Fourth, the binary interactions are probably changed by the greater compactness of low Z stars on the MS. Furthermore, the first generation stars below $40 \mathrm{M}_{\odot}$ do not evolve to the RSG stage. De Mink et al. (2008) show that as Z decreases, mass transfer is more likely to take place after the ignition of He burning (case C) and for the very low- $Z$ stars, which do not reach the RSG stage, only the closest binaries would still interact.

Finally, the first stars are thought to be more massive than solar-metallicity stars (Bromm \& Larson 2004, Schneider et al. 2006). Note that other studies suggest that both very massive and low-mass stars may form at $Z=0$ (Nakamura \& Umemura 2001). Since mass loss is expected to be very low at very low metallicities, the logical deduction from these two arguments is that a large fraction of the first stars were very massive at their death $\left(>100 \mathrm{M}_{\odot}\right)$ and therefore lead to the production of pair-creation supernovae (PCSNe). Unfortunately, the first massive stars died a long time ago and will probably never be detected directly (see however Scannapieco et al. 2005, Tornatore et al. 2007). There are nevertheless indirect observational constraints on the first stars coming from observations of the most metal-poor halo stars (Beers \& Christlieb 2005). These observations do not show the peculiar chemical signature of PCSNe (strong oddeven effects and low zinc, see Heger \& Woosley 2002). This probably means that at most only a few of these very massive stars $\left(>100 \mathrm{M}_{\odot}\right)$ formed or that they lost a lot of mass even though their initial metal content was very low as discussed in the next section. Although there is no signature of PCSNe at very low Z, they might occur in our local Universe (Smith et al. 2007, Langer et al. 2007, Woosley et al. 2007).

The topic of low Z stellar evolution is not new (see for example Chiosi 1983, El Eid et al. 1983, Carr et al. 1984, Arnett 1996). The observations of extremely metal-poor stars (Beers \& Christlieb 2005) have however greatly increased the interest in very metalpoor stars. There are many recent works studying the evolution of metal-free (or almost) massive (Heger \& Woosley 2002, Limongi \& Chieffi 2005, Umeda \& Nomoto 2005, Meynet et al. 2006), intermediate mass (Siess et al. 2002, Herwig 2004, Suda et al. 2004, GilPons et al. 2005) and low mass (Picardi et al. 2004, Weiss et al. 2004) stars in an attempt to explain the origin of the surface abundances observed. The fate of non-rotating massive single stars at low Z is summarised in Heger et al. (2003) and several groups have calculated the corresponding stellar yields (Heger \& Woosley 2002, Chieffi \& Limongi 2004, Tominaga et al. 2007). 


\section{Rotation, internal mixing and mass loss}

Massive star models including the effects of both mass loss and especially rotation better reproduce many observables around solar $Z$ (See contributions by Meynet and Maeder in this volume). For example, models with rotation allow chemical surface enrichments already on the main sequence (MS), whereas without the inclusion of rotation, self-enrichments are only possible during the RSG stage (Heger \& Langer 2000, Meynet \& Maeder 2000). Rotating star models also better reproduce the WR/O ratio and also the ratio of type $\mathrm{Ib}+\mathrm{Ic}$ to type II supernova as a function of metallicity compared to non-rotating models, which underestimate these ratios (Meynet \& Maeder 2005). The models at very low $Z$ presented here use the same physical ingredients as the successful solar $Z$ models. The value of $300 \mathrm{~km} \mathrm{~s}^{-1}$ used as the initial rotation velocity at solar metallicity corresponds to an average velocity of about $220 \mathrm{~km} \mathrm{~s}^{-1}$ on the main sequence (MS) which is close to the average observed value. See for instance Fukuda (1982) for one of the first surveys and the list in Meynet's contribution in this volume for the most recent surveys. It is unfortunately not possible to observe very low $Z$ massive stars and measure their rotational velocity since they all died a long time ago. Higher observed ratio of Be to B stars in the Magellanic clouds compared to our Galaxy (Maeder et al. 1999 ) could point out to the fact the stars rotate faster at lower metallicities. Also a low- $Z$ star having the same ratio of surface velocity to critical velocity, $v / v_{\text {crit }}$ (where $v_{\text {crit }}$ is the velocity for which the centrifugal force balances the gravitational force) as a solar- $Z$ star has a higher surface rotation velocity due to its smaller radius (one quarter of $Z_{\odot}$ radius for a very low $Z 20 \mathrm{M}_{\odot}$ star). In the models presented below, the initial ratio $v / v_{\text {crit }}$ is the same or slightly higher than for solar Z (see Hirschi 2007 for more details). This corresponds to initial surface velocities in the range of $600-800 \mathrm{~km} \mathrm{~s}^{-1}$. These fast initial rotation velocities are supported by chemical evolution models of Chiappini et al. (2006b) discussed in the next section. The mass loss prescriptions used in the Geneva stellar evolution code are described in detail in Meynet \& Maeder (2005). In particular, the mass loss rates depend on metallicity as $\dot{M} \sim\left(Z / Z_{\odot}\right)^{0.5}$, where $Z$ is the mass fraction of heavy elements at the surface of the star.

How do rotation induced processes vary with metallicity? The surface layers of massive stars usually accelerate due to internal transport of angular momentum from the core to the envelope. Since at low $Z$, stellar winds are weak, this angular momentum dredged up by meridional circulation remains in the star, and the star more easily reaches critical rotation. At the critical limit, matter can easily be launched into a keplerian disk which probably dissipates under the action of the strong radiation pressure of the star.

The efficiency of meridional circulation (dominating the transport of angular momentum) decreases towards lower $\mathrm{Z}$ because the Gratton-Öpik term of the vertical velocity of the outer cell is proportional to $1 / \rho$. On the other hand, shear mixing (dominating the mixing of chemical elements) is more efficient at low $Z$. Indeed, the star is more compact and therefore the gradients of angular velocity are larger and the mixing timescale (proportional to the square of the radius) is shorter. This leads to stronger internal mixing of chemical elements at low Z (Meynet \& Maeder 2002).

Figure 2 shows the evolution of the convective zones in a rotating and a non-rotating $20 \mathrm{M}_{\odot}$ models at $Z=10^{-8}$. The history of convective zones (in particular the convective zones associated with shell $\mathrm{H}$ burning and core He burning) is strongly affected by rotation induced mixing. The most important rotation induced mixing takes place while helium is burning inside a convective core. Primary carbon and oxygen are mixed outside of the convective core into the H-burning shell. Once the enrichment is strong enough, the H-burning shell is boosted (the CNO cycle depends strongly on the carbon and 

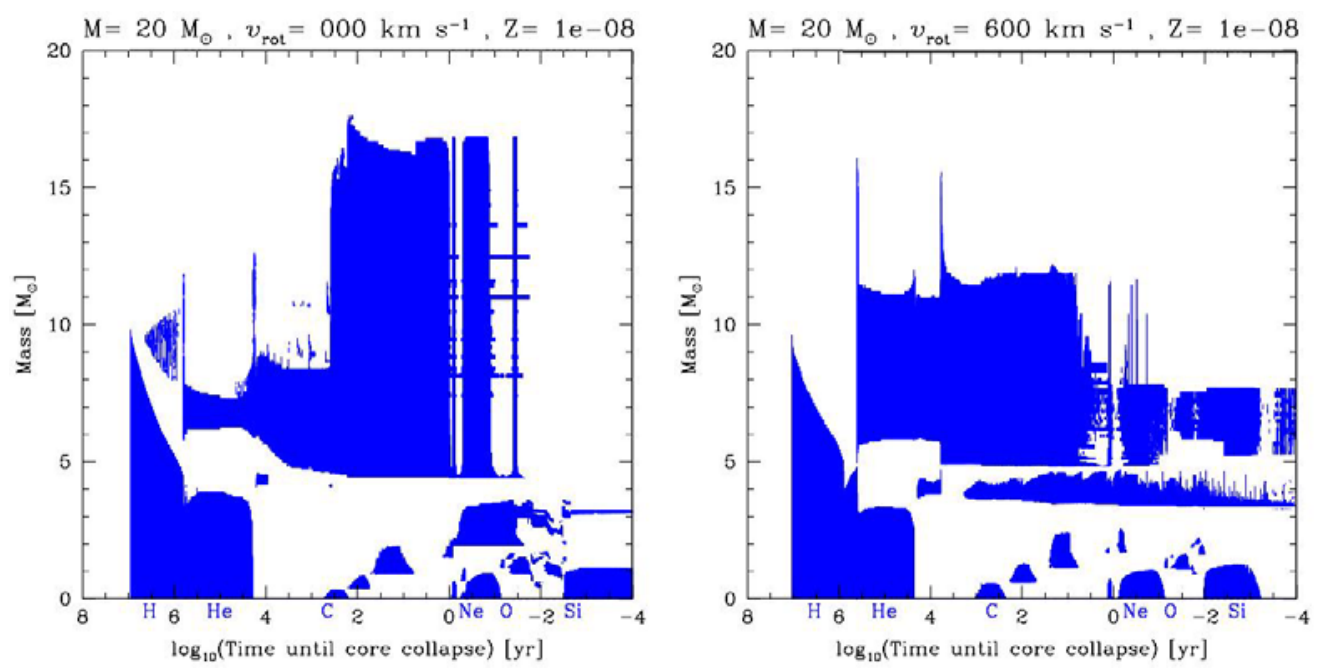

Figure 2. Structure evolution diagram for the non-rotating (left) and rotating (right) $20 \mathrm{M}_{\odot}$ models at $Z=10^{-8}$. Black (coloured) areas correspond to convective zones along the Lagrangian mass coordinate as a function of the time left until the core collapse. The burning stage abbreviations are given below the time axis. Rotation strongly affects shell $\mathrm{H}$ burning and core $\mathrm{He}$ burning.

oxygen mixing at such low initial metallicities). The shell then becomes convective and leads to an important primary nitrogen production. In response to the shell boost, the core expands and the convective core mass decreases. At the end of He burning, the CO core is less massive than in the non-rotating model (see Fig. 2). Additional convective and rotational mixing brings the primary CNO to the surface of the star. This has consequences for the stellar yields. The yield of ${ }^{16} \mathrm{O}$ being closely correlated with the mass of the $\mathrm{CO}$ core, it is therefore reduced due to the strong mixing. At the same time the carbon yield is slightly increased. The relatively "low" oxygen yields and "high" carbon yields are produced over a large mass range at $Z=10^{-8}$ (Hirschi 2007). This could be an explanation for the possible high $[\mathrm{C} / \mathrm{O}]$ ratio observed in the most metal-poor halo stars (ratio between the surface abundances of carbon and oxygen relative to solar; see Fig. 14 in Spite et al. 2005).

Models of metal-free stars including the effect of rotation (Ekström et al. 2006) show that stars may lose up to $10 \%$ of their initial mass due to the star rotating at its critical limit (also called break-up limit). The mass loss due to the star reaching the critical limit is non-negligible but not important enough to change drastically the fate of the metal-free stars. The situation is very different at very low but non-zero metallicity (Meynet et al. 2006, Hirschi 2007). The total mass of an $85 \mathrm{M}_{\odot}$ model at $Z=10^{-8}$ is shown in Fig. 3 by the top solid line. This model, like metal-free models, loses around $5 \%$ of its initial mass when its surface reaches break-up velocities in the second part of the MS. At the end of core $\mathrm{H}$ burning, the core contracts and the envelope expands, thus decreasing the surface velocity and its ratio to the critical velocity. The mass loss rate becomes very low again until the star crosses the HR diagram and reaches the RSG stage. In the cooler part of the $\mathrm{H}-\mathrm{R}$ diagram, the mass loss becomes very important. This is due to the dredge-up by the convective envelope of CNO elements to the surface increasing its overall metallicity. The total metallicity, $Z$, is used in this model (including CNO elements) for the metallicity dependence of the mass loss. Therefore depending on how much CNO is brought up to the surface, the mass loss becomes very large again. The CNO brought to the surface 
comes from primary $\mathrm{C}$ and $\mathrm{O}$ produced in the He-burning region and from primary $\mathrm{N}$ produced in the H-burning one.

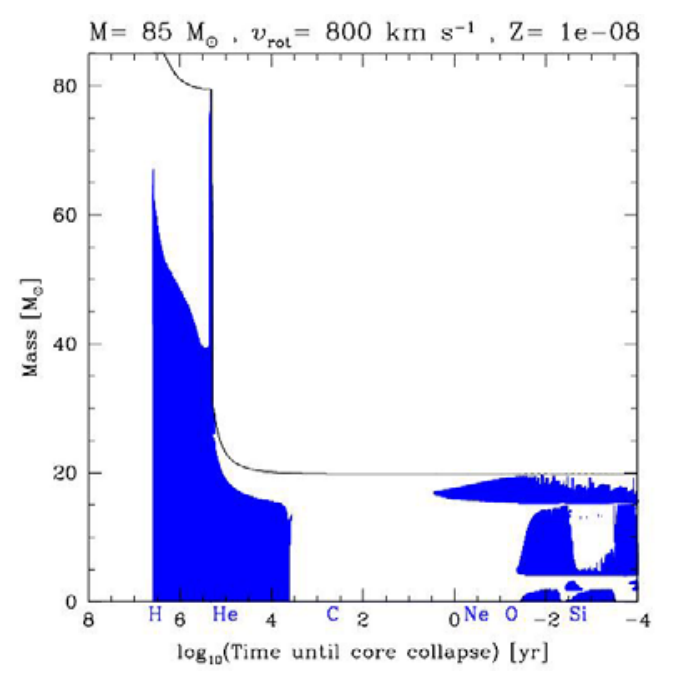

Figure 3. Structure evolution diagram (same as Fig. 2) for a $85 \mathrm{M}_{\odot}$ model with $v_{\text {ini }}=800$ $\mathrm{km} \mathrm{s}^{-1}$ at $Z=10^{-8}$. The top solid line shows the total mass of the star. A strong mass loss during the RSG stage removes a large fraction of total mass of the star.

Could such low- $Z$ stars undergo dust-driven winds? For this to occur, the surface effective temperature needs to be low enough (usually $\log \left(\mathrm{T}_{\text {eff }}\right)<3.6$ ) and carbon needs to be more abundant than oxygen. This last condition is fulfilled in our $85 \mathrm{M}_{\odot}$ model. However, it is presently unclear if: 1) Extremely low- $Z$ stars reach such low effective temperatures. This depends on the opacity and the opacity tables used in our calculations did not account for the non-standard mixture of metals (high CNO and low iron abundance, see Marigo 2002 for possible effects). 2) At such low $Z$, enough metal is present to allow dust formation. Indeed, nucleation seeds (probably involving titanium) are necessary to form C-rich dust. There may also be other important types of wind, like chromospheric activity-driven, pulsation-driven, thermally-driven or continuum-driven winds.

The fate of rotating stars at very low $\mathrm{Z}$ is therefore probably the following:

- $M<40 \mathrm{M}_{\odot}$ : Mass loss is insignificant and matter is only ejected into the ISM during the SN explosion (see contributions by Nomoto et al, Limongi et al and Fröhlich et al in this volume), which could be very energetic if fast rotation is still present in the core at the core collapse.

- $40 \mathrm{M}_{\odot}<M<60 \mathrm{M}_{\odot}$ : Mass loss (at critical rotation and in the RSG stage) removes $10-20 \%$ of the initial mass of the star. The star probably dies as a black hole without a SN explosion and therefore the feedback into the ISM is only due to stellar winds, which are slow.

- $M>60 \mathrm{M}_{\odot}$ : A strong mass loss removes a significant amount of mass and the stars enter the WR phase. These stars therefore die as type Ib/c SNe and possibly as GRBs.

\section{Nucleosynthesis and galactic chemical evolution}

Rotation induced mixing leads to the production of primary nitrogen, ${ }^{13} \mathrm{C}$ and ${ }^{22} \mathrm{Ne}$. In this section, we compare the chemical composition of our models with carbon-rich EMP 
stars and include our stellar yields in a galactic chemical evolution (GCE) model and compare the GCE model with observations of EMP stars.

\subsection{The most metal-poor star known to date, HE1327-2326}

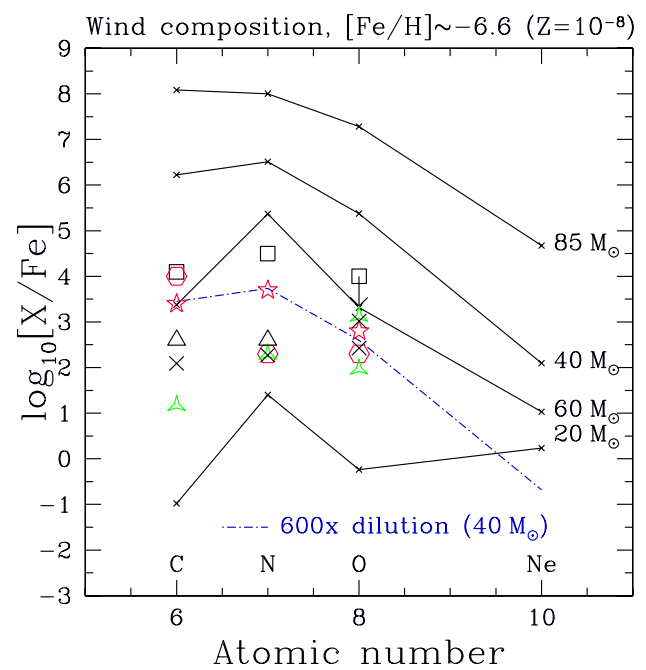

Figure 4. Composition in $[\mathrm{X} / \mathrm{Fe}]$ of the stellar wind for the $Z=10^{-8}$ models (solid lines). For HE1327-2326 (stars), the best fit for the CNO elements is obtained by diluting the composition of the wind of the $40 \mathrm{M}_{\odot}$ model by a factor 600 (see Hirschi 2007 for more details).

Significant mass loss in very low- $Z$ massive stars offers an interesting explanation for the strong enrichment in CNO elements of the most metal-poor stars observed in the halo of the galaxy (see Meynet et al. 2006, Hirschi 2007). The most metal-poor star known to date, HE1327-2326 (Frebel et al. 2006) is characterised by very high N, C and O abundances, high $\mathrm{Na}, \mathrm{Mg}$ and $\mathrm{Al}$ abundances, an s-process ( $\mathrm{sr}$ ) enrichment and depleted lithium. The star is not evolved so has not had time to bring self-produced CNO elements to its surface and is most likely a subgiant. By using one or a few SNe and using a very large mass cut, Limongi et al. (2003) and Iwamoto et al. (2005) are able to reproduce the abundance of most elements. However they are not able to reproduce the nitrogen surface abundance of HE1327-2326 without rotational mixing. The abundance pattern observed at the surface of that star present many similarities with the abundance pattern obtained in the winds of very metal poor fast rotating massive star models. HE13272326 may therefore have formed from gas, which was mainly enriched by stellar winds of rotating very low metallicity stars. In this scenario, a first generation of stars (PopIII) pollutes the interstellar medium to very low metallicities $([\mathrm{Fe} / \mathrm{H}] \sim-6)$. Then a PopII.5 star (Hirschi 2005) like the $40 \mathrm{M}_{\odot}$ model calculated here pollutes (mainly through its wind) the interstellar medium out of which HE1327-2326 forms. This would mean that HE1327-2326 is a third generation star. In this scenario, the CNO abundances are well reproduced, in particular that of nitrogen, which according to the latest values for a subgiant (see Frebel et al. 2006) is 0.9 dex higher in $[\mathrm{X} / \mathrm{Fe}]$ than oxygen. This is shown in Fig. 4 where the abundances of HE1327-2326 are represented by the red stars and the best fit is obtained by diluting the composition of the wind of the $40 \mathrm{M}_{\odot}$ model by a factor 600 . When the $\mathrm{SN}$ contribution is added, the $[\mathrm{X} / \mathrm{Fe}]$ ratio is usually lower for nitrogen than for oxygen. It is interesting to note that the very high CNO yields of the 


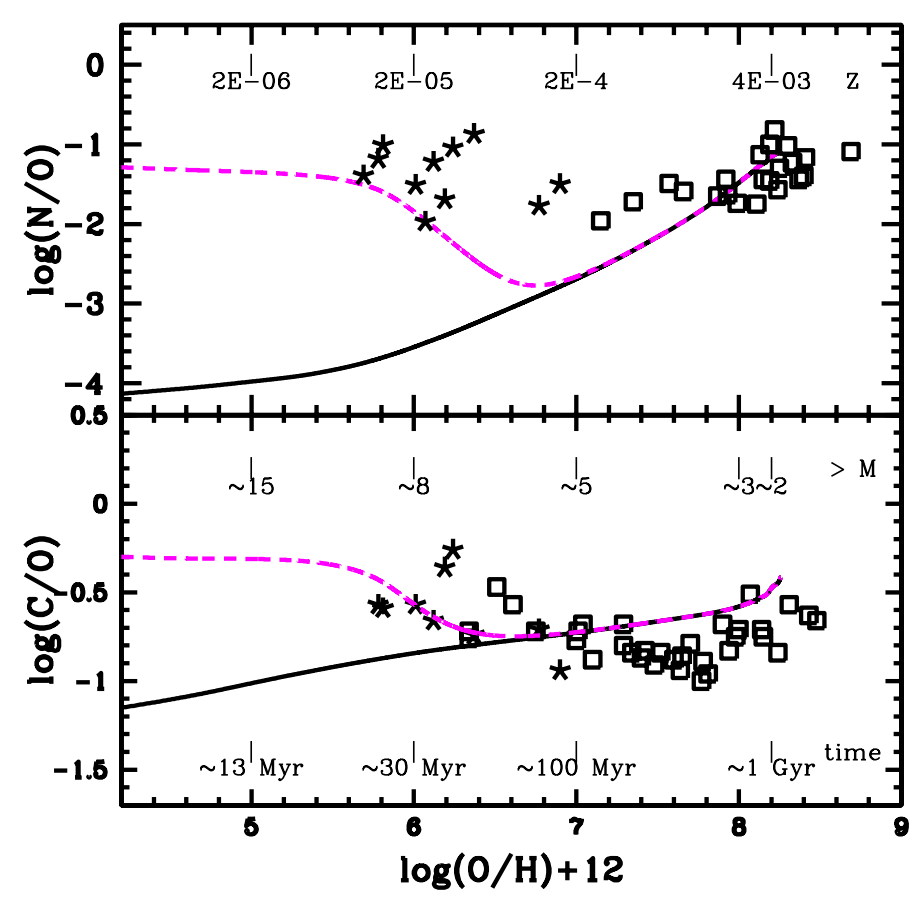

Figure 5. Chemical evolution model predictions of the N/O and $\mathrm{C} / \mathrm{O}$ evolution, in the galactic halo, for different stellar evolution inputs. The solid curves show the predictions of a model without fast rotators at low metallicities. The dashed lines show the effect of including a population of fast rotators at low metallicities. For the data see Chiappini et al. (2006b) and references therein.

$40 \mathrm{M}_{\odot}$ stars brings the total metallicity $Z$ above the limit for low mass star formation obtained in Bromm \& Loeb (2003).

\subsection{Primary nitrogen and ${ }^{13} \mathrm{C}$}

The high N/O plateau values observed at the surface of very metal poor halo stars require very efficient sources of primary nitrogen. Rotating massive stars can inject in a short timescale large amount of primary $\mathrm{N}$. They are therefore very good candidates to explain the N/O plateau observed at very low metallicity. According to the heuristic model of Chiappini et al. (2005), a primary nitrogen production of about $0.15 \mathrm{M}_{\odot}$ per star is necessary. Upon the inclusion of the stellar yields including the effects of fast rotation at $\mathrm{Z}=10^{-8}$ in a chemical evolution model for the galactic halo with infall and outflow, both high $\mathrm{N} / \mathrm{O}$ and $\mathrm{C} / \mathrm{O}$ ratios are obtained in the very metal-poor metallicity range in agreement with observations (see details in Chiappini et al. 2006a). This model is shown in Fig. 5 (dashed magenta curve). In the same figure, a model computed without fast rotators (solid black curve) is also shown. Fast rotation enhances the nitrogen production by $\sim 3$ orders of magnitude. These results also offer a natural explanation for the large scatter observed in the $\mathrm{N} / \mathrm{O}$ abundance ratio of normal metal-poor halo stars: given the strong dependency of the nitrogen yields on the rotational velocity of the star, we expect a scatter in the $\mathrm{N} / \mathrm{O}$ ratio which could be the consequence of the distribution of the stellar rotational velocities as a function of metallicity.

As explained above, the strong production of primary nitrogen is linked to a very active H-burning shell and therefore a smaller helium core. As a consequence, less carbon is 


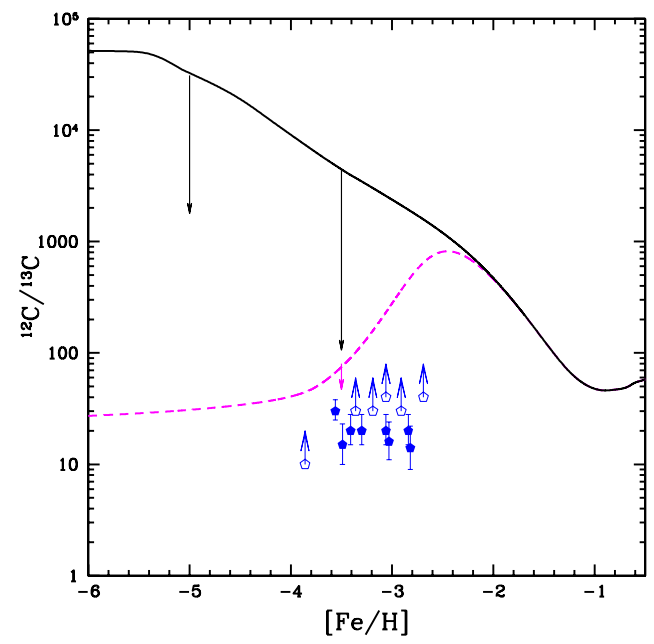

Figure 6. See a detailed description of this Fig. in Chiappini et al. (2008)

turned into oxygen, producing high $\mathrm{C} / \mathrm{O}$ ratios. Although the abundance data for $\mathrm{C} / \mathrm{O}$ is still very uncertain, a $\mathrm{C} / \mathrm{O}$ upturn at low metallicities is suggested by observations (see Asplund 2005 and references therein). Note that this upturn is now also observed in very metal poor DLA systems (see the paper by M. Pettini in this volume).

In addition, stellar models of fast rotators have a great impact on the evolution of the ${ }^{12} \mathrm{C} /{ }^{13} \mathrm{C}$ ratio at very low metallicities (Chiappini et al. 2008). In this case, we predict that, if fast rotating massive stars were common phenomena in the early Universe, the primordial interstellar medium of galaxies with a star formation history similar to the one inferred for our galactic halo should have ${ }^{12} \mathrm{C} /{ }^{13} \mathrm{C}$ ratios between $30-300$. Without fast rotators, the predicted ${ }^{12} \mathrm{C} /{ }^{13} \mathrm{C}$ ratios would be $\sim 4500$ at $[\mathrm{Fe} / \mathrm{H}]=-3.5$, increasing to $\sim 31000$ at around $[\mathrm{Fe} / \mathrm{H}]=-5.0$ (see Fig. 6 ).

Current data on EMP giant normal stars in the galactic halo (Spite et al. 2006) agree better with chemical evolution models including fast rotators. The expected difference in the ${ }^{12} \mathrm{C} /{ }^{13} \mathrm{C}$ ratios, after accounting for the effects of the first dredge-up (indicated by the arrows in Fig. 6), between our predictions with/without fast rotators is of the order of a factor of 2-3. However, larger differences (a factor of $\sim 60-90$ ) are expected for giants at $[\mathrm{Fe} / \mathrm{H}]=-5$ or turnoff stars already at $[\mathrm{Fe} / \mathrm{H}]=-3.5$. To test our predictions, challenging measurements of the ${ }^{12} \mathrm{C} /{ }^{13} \mathrm{C}$ in more extremely metal-poor giants and turnoff stars are required.

\subsection{Primary ${ }^{22} \mathrm{Ne}$ and $s$ process at low $Z$}

Models at $Z=10^{-8}$ show a production of primary ${ }^{22} \mathrm{Ne}$ during He burning. We also started calculating models at different $\mathrm{Z}$ to determine over which $\mathrm{Z}$ range the primary production of ${ }^{22} \mathrm{Ne}$ and also ${ }^{14} \mathrm{~N}$ is important. In Fig. 7, we show the properties of a 20 $\mathrm{M}_{\odot}$ model at $Z=10^{-6}$ up to the end of He burning. Around $0.5 \%$ (in mass fraction) of ${ }^{22} \mathrm{Ne}$ is burnt during core He burning and therefore leads to a significant neutron release. Studies are underway to determine how much s process can be produced in these models. 

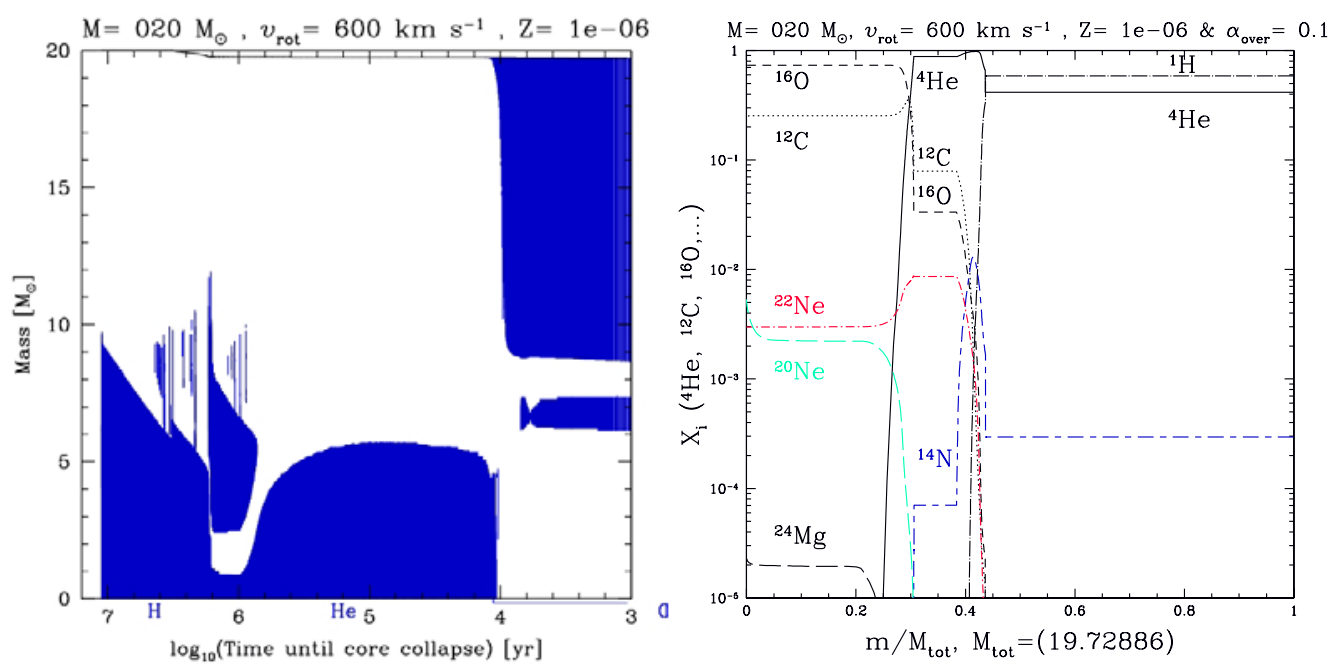

Figure 7. Left: Structure evolution diagram (see description of Fig. 2) for a rotating $20 \mathrm{M}_{\odot}$ models at $Z=10^{-6}$ during $\mathrm{H}$ - and He-burning phases. Right: Chemical composition at the end of core He burning. Just above the core, one sees that the maximum abundance of ${ }^{22} \mathrm{Ne}$ is around $1 \%$ in mass fraction and at the end of core He burning, around $0.5 \%$ is burnt in the core, providing plenty of neutrons for s process.

\section{Magnetic fields and GRBs}

In this last section, we discuss the impact of magnetic fields. Models of rotating stars, which do not include the effect of magnetic fields predict gamma-ray bursts (GRBs) at almost all Z (Hirschi et al. 2005, Hirschi 2007). However, they also overestimate the initial rotation rate of pulsars. The inclusion of the effects of magnetic fields according to Spruit (2002) allows a better reproduction of the initial pulsar periods (Heger et al. 2005). Along with gravity waves, magnetic fields are also one possible cause for the flat rotation profile of the Sun (Eggenberger et al. 2005). Although it becomes much harder for the core to retain enough angular momentum until the core collapse, there is still an evolutionary scenario, the so-called chemically homogeneous evolution, leading to the production of fast rotating cores at the pre-SN stage and therefore enabling MHD explosions and GRBs (see Yoon et al. 2006, Woosley \& Heger 2006 and also Yoon's contribution in this volume). The theoretical GRB event rates obtained by Yoon et al. (2006) are in good agreement with observations apart from the upper metallicity limit, which is lower than the observed one (see contribution by Stanek in this volume). Yoon et al. (2006) also predict that at $Z=10^{-5}$, a large fraction of massive stars are GRB progenitors. We have calculated $40 \mathrm{M}_{\odot}$ models at $Z=10^{-5}$ with $v_{\text {ini }} / v_{\text {crit }}=0.59$ and at $Z=10^{-8}$ with $v_{\text {ini }} / v_{\text {crit }}=0.55$. The model at $Z=10^{-5}$ confirms the possibility of producing GRBs down to very low Z. However, the model at $Z=10^{-8}$ does not rotate fast enough to evolve chemically homogeneously. The difficulty of the very low $\mathrm{Z}$ models to evolve chemically homogeneously is due to the weakening of the meridional circulation. Indeed, in models including magnetic fields, meridional circulation becomes the dominant term for the mixing of chemical species (see Maeder \& Meynet 2005). The meridional circulation becomes weaker at low $Z$ because the meridional currents are less efficient in a denser medium, which is the case since low- $Z$ stars are more compact. This means that not all stars in the first stellar generations will produce GRBs in this way. Finally, it is interesting to note that the presence of magnetic fields in metal-free stars may enhance mass loss significantly (see contribution by Ekström et al in this volume). 


\section{Conclusions and outlook}

The inclusion of the effects of rotation changes significantly the simple picture in which stellar evolution at low $\mathrm{Z}$ is just stellar evolution without mass loss. A strong mixing is induced between the helium and hydrogen burning layers leading to a significant production of primary ${ }^{14} \mathrm{~N},{ }^{13} \mathrm{C}$ and ${ }^{22} \mathrm{Ne}$. Rotating stellar models also predict a strong mass loss during the RSG stage for stars more massive than $60 \mathrm{M}_{\odot}$. The chemical composition of the stellar winds is compatible with the CNO abundance observed in the most metal-poor star known to date, HE1327-2326. GCE models including the stellar yields of these rotating star models are able to better reproduce the early evolution of $\mathrm{N} / \mathrm{O}, \mathrm{C} / \mathrm{O}$ and ${ }^{12} \mathrm{C} /{ }^{13} \mathrm{C}$ in our galaxy. These models predict a large neutron release during core $\mathrm{He}$ burning and thus a strong possibility of an s process at very low Z. These models predict the formation of $\mathrm{WR}$ and type $\mathrm{Ib} / \mathrm{c} \mathrm{SNe}$ down to almost $\mathrm{Z}=0$. The inclusion of magnetic fields slows down the core of the stars and therefore reduces the probability of producing GRBs at metallicities around that of the Magellanic Clouds but GRBs are still predicted from single star models down to very low $\mathrm{Z}$.

Large surveys of EMP stars (SEGUE, OZ surveys), of GRBs and SNe (Swift and GLAST satellites) and of massive stars (e. g. VLT FLAMES survey) are underway and will bring more information and constraints on the evolution of massive stars at low $\mathrm{Z}$. On the theoretical side, more models are necessary to fully understand and study the complex interplay between rotation, magnetic fields, mass loss and binary interactions at different metallicities. Large grids of models at low $\mathrm{Z}$ will have many applications, for example to study the evolution of massive stars and their feedback in high redshift objects like Lyman-break galaxies and damped Ly-alpha systems.

\section{Acknowledgements}

R. Hirschi acknowledges financial support from EPSAM, from the organizers (IAU Grant), and from the Royal Society (Conference Grant round 2007/R3).

\section{References}

Arnett, D. 1996, in: H. L. Morrison \& A. Sarajedini (eds.), Formation of the Galactic Halo...Inside and Out, (San Francisco: ASP) ASP Conf. Ser., 92, 337

Asplund, M. 2005, ARAEAA, 43, 481

Beers, T. C. \& Christlieb, N. 2005, ARA\&A, 43, 531

Bromm, V. \& Larson, R. B. 2004, ARAEAA, 42, 79

Bromm, V. \& Loeb, A. 2003, Nature, 425, 812

Carr, B. J., Bond, J. R., \& Arnett, W. D. 1984, ApJ, 277, 445

Chiappini, C., Matteucci, F., \& Ballero, S. K. 2005, A\& $A, 437,429$

Chiappini, C., Hirschi, R., Matteucci, F., et al. 2006a, in: Nuclei in the Cosmos IX, CERN, PoS(NIC-IX) 080

Chiappini, C., Hirschi, R., Meynet, G., et al. 2006b, A\& $A$, 449, L27

Chiappini, C., Ekström, S., Meynet, G., et al. 2008, A\&A, 479, L9

Chieffi, A. \& Limongi, M. 2004, ApJ, 608, 405

Chiosi, C. 1983, Memorie della Societa Astronomica Italiana, 54, 251

de Mink, S. E., Pols, O. R., \& Yoon, S. C. 2008, in: T. Abel, A. Heger \& B. O'Shea (eds.), First Stars III (New York: AIP), AIP Conf Proc., in press (arXiv0710.1010)

Eggenberger, P., Maeder, A., \& Meynet, G. 2005, A\&A, 440, L9

Ekström, S., Meynet, G., \& Maeder, A. 2006, in: H. J.G. L.M. Lamers, N. Langer, T. Nugis, \& K. Annuk (eds.), Stellar Evolution at Low Metallicity: Mass Loss, Explosions, Cosmology (San Francisco: ASP) ASP Conf. Ser., 353, 141

Ekström, S., Meynet, G., \& Maeder, A. 2008, in: T. Abel, A. Heger \& B. O'Shea (eds.), First Stars III, (New York: AIP), AIP Conf. Proc., in press (arXiv:0709.0202) 
El Eid, M. F., Fricke, K. J., \& Ober, W. W. 1983, A\&A, 119, 54

Ferrarotti, A. S. \& Gail, H.-P. 2006, $A \mathscr{E} A$, 447, 553

Frebel, A., Christlieb, N., Norris, J. E., et al. 2006, ApJ, 638, L17

Fukuda, I. 1982, PASP, 94, 271

Gil-Pons, P., Suda, T., Fujimoto, M. Y., \& García-Berro, E. 2005, A\&\&A, 433, 1037

Heger, A. \& Langer, N. 2000, ApJ, 544, 1016

Heger, A. \& Woosley, S. E. 2002, ApJ, 567, 532

Heger, A., Fryer, C. L., Woosley, S. E., et al. 2003, ApJ, 591, 288

Heger, A., Woosley, S. E., \& Spruit, H. C. 2005, ApJ, 626, 350

Herwig, F. 2004, ApJS, 155, 651

Hirschi, R. 2005, in: V. Hill, P. François \& F. Primas (eds.), From Lithium to Uranium: Elemental Tracers of Early Cosmic Evolution (Cambridge: Cambridge University Press), Proc. IAU Symp, 228, 331

Hirschi, R. 2007, A\& A, 461, 571

Hirschi, R., Meynet, G., \& Maeder, A. 2005, A\&A A, 443, 581

Höfner, S. \& Andersen, A. C. 2007, A\& A, 465, L39

Iwamoto, N., Umeda, H., Tominaga, N., et al. 2005, Science, 309, 451

Kudritzki, R. P. 2002, ApJ, 577, 389

Kudritzki, R.-P. \& Puls, J. 2000, ARA\&A, 38, 613

Langer, N., Norman, C. A., de Koter, A., et al. 2007, A\& A, 475, L19

Limongi, M. \& Chieffi, A. 2005, in: V. Hill, P. François \& F. Primas (eds.), From Lithium to Uranium: Elemental Tracers of Early Cosmic Evolution (Cambridge: Cambridge University Press), Proc. IAU Symp, 228, 303

Limongi, M., Chieffi, A., \& Bonifacio, P. 2003, ApJ, 594, L123

Maeder, A., Grebel, E. K., \& Mermilliod, J.-C. 1999, A\&A, 346, 459

Maeder, A. \& Meynet, G. 2005, A\&A A, 440, 1041

Marigo, P. 2002, A\&A A, 387, 507

Meynet, G. \& Maeder, A. 2000, $A \mathscr{E} A, 361,101$

Meynet, G. \& Maeder, A. 2002, $A \mathscr{G} A, 390,561$

Meynet, G. \& Maeder, A. 2005, $A \mathscr{G} A, 429,581$

Meynet, G., Ekström, S., \& Maeder, A. 2006, A\&A, 447, 623

Mokiem, M. R., de Koter, A., Vink, J. S., et al. 2007, A\&A, 473, 603

Nakamura, F. \& Umemura, M. 2001, ApJ, 548, 19

Nieuwenhuijzen, H., \& de Jager, C. 1990, A\&AA, 231, 134

Picardi, I., Chieffi, A., Limongi, M., et al. 2004, ApJ, 609, 1035

Scannapieco, E., Madau, P., Woosley, S., et al. 2005, ApJ, 633, 1031

Schneider, R., Omukai, K., Inoue, A. K., \& Ferrara, A. 2006, MNRAS, 369, 1437

Siess, L., Livio, M., \& Lattanzio, J. 2002, ApJ, 570, 329

Smith, N., Gehrz, R. D., Hinz, P. M., et al. 2003, AJ, 125, 1458

Smith, N., Li, W., Foley, R. J., et al. 2007, ApJ, 666, 1116

Spite, M., Cayrel, R., Plez, B., et al. 2005, A\&BA, 430, 655

Spite, M., Cayrel, R., Hill, V., et al. 2006, A\&BA, 455, 291

Spruit, H. C. 2002, A\&BA, 381, 923

Suda, T., Aikawa, M., Machida, M. N., et al. 2004, ApJ, 611, 476

Tominaga, N., Umeda, H., \& Nomoto, K. 2007, ApJ, 660, 516

Tornatore, L., Ferrara, A., \& Schneider, R. 2007, MNRAS, 382, 945

Umeda, H. \& Nomoto, K. 2005, ApJ, 619, 427

van Loon, J. T. 2000, A\& A, 354, 125

van Loon, J. T. 2006, in: H. J.G. L.M. Lamers, N. Langer, T. Nugis, \& K. Annuk (eds.), Stellar Evolution at Low Metallicity: Mass Loss, Explosions, Cosmology (San Francisco: ASP), ASP Conf. Ser., 353, 211

van Loon, J. T., Cioni, M.-R. L., Zijlstra, A. A., \& Loup, C. 2005, A\&A, 438, 273

Vink, J. S. \& de Koter, A. 2005, A\&A, 442, 587

Vink, J. S., de Koter, A., \& Lamers, H. J.G. L.M. 2000, AछA, 362, 295

Vink, J. S., de Koter, A., \& Lamers, H. J.G. L.M. 2001, A\&A, 369, 574 
Weiss, A., Schlattl, H., Salaris, M., \& Cassisi, S. 2004, A\& A, 422, 217

Woosley, S. E. \& Heger, A. 2006, ApJ, 637, 914

Woosley, S. E., Blinnikov, S., \& Heger, A. 2007, Nature, 450, 390

Yoon, S.-C., Langer, N., \& Norman, C. 2006, A\&AA, 460, 199

\section{Discussion}

LEITHERER: Your models seem to have large mass loss, even at very low Z. Does this imply that the winds are dense enough to hide the hot core? Then we would not expect an extremely hard ionizing spectrum and little observable nebular He II $\lambda 1640$, a possible telltale sign of the first generation of stars.

HiRSCHI: Mass loss takes place after the main sequence, so the ionizing flux can still be produced during the main sequence.

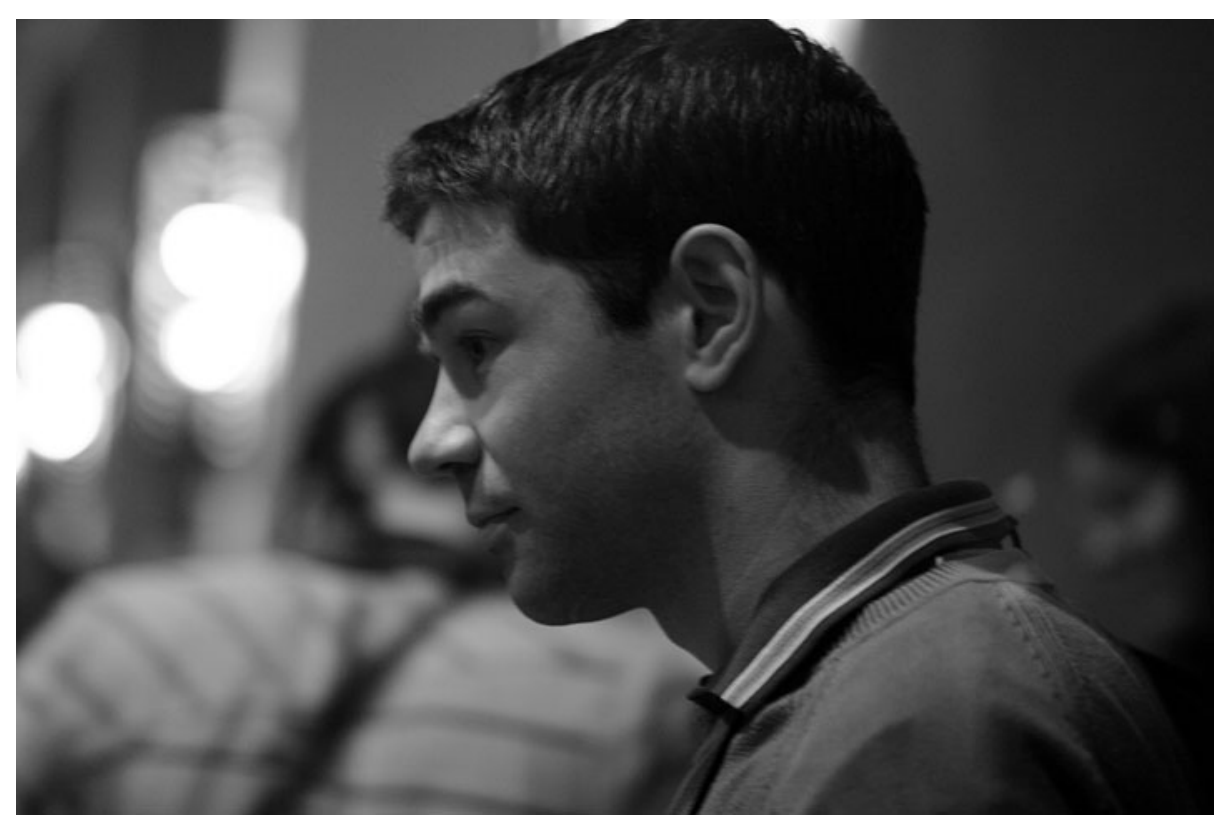

Raphael Hirschi. 


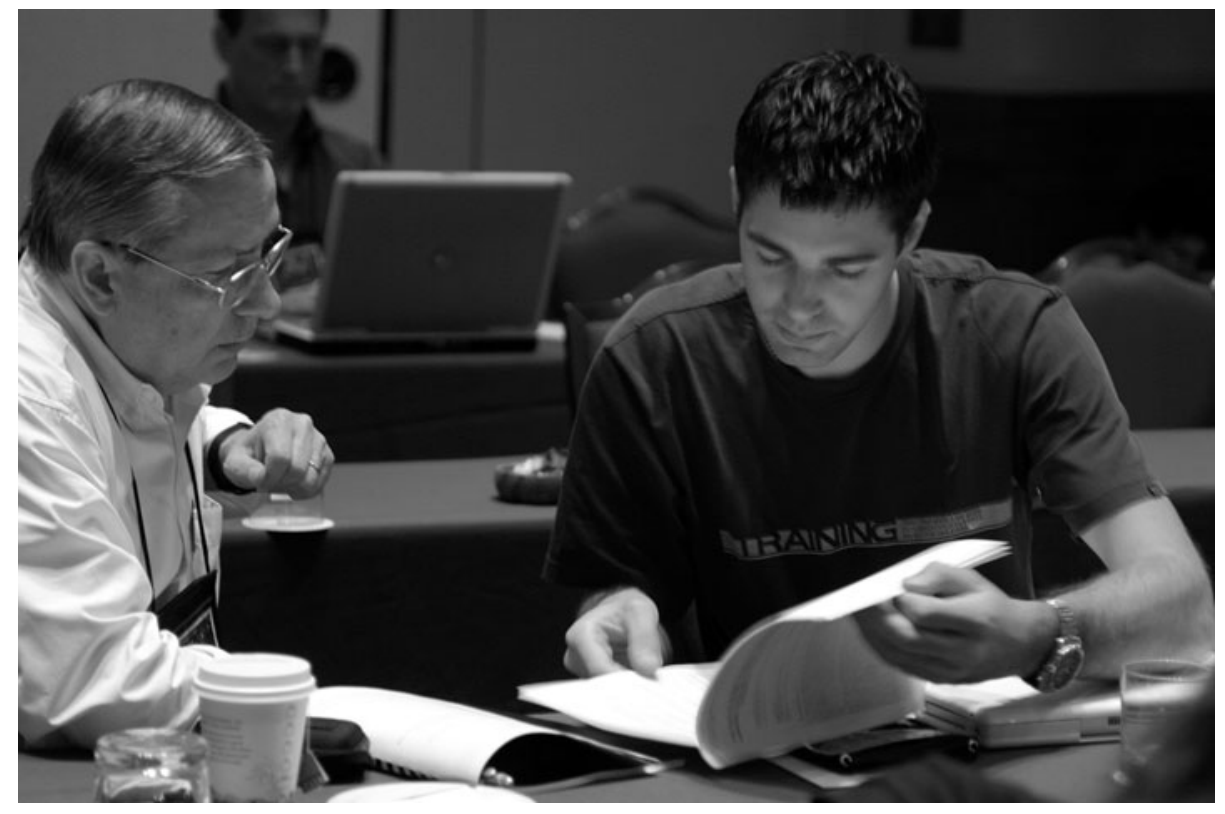

Nolan Walborn (left) and Ian Hunter (right).

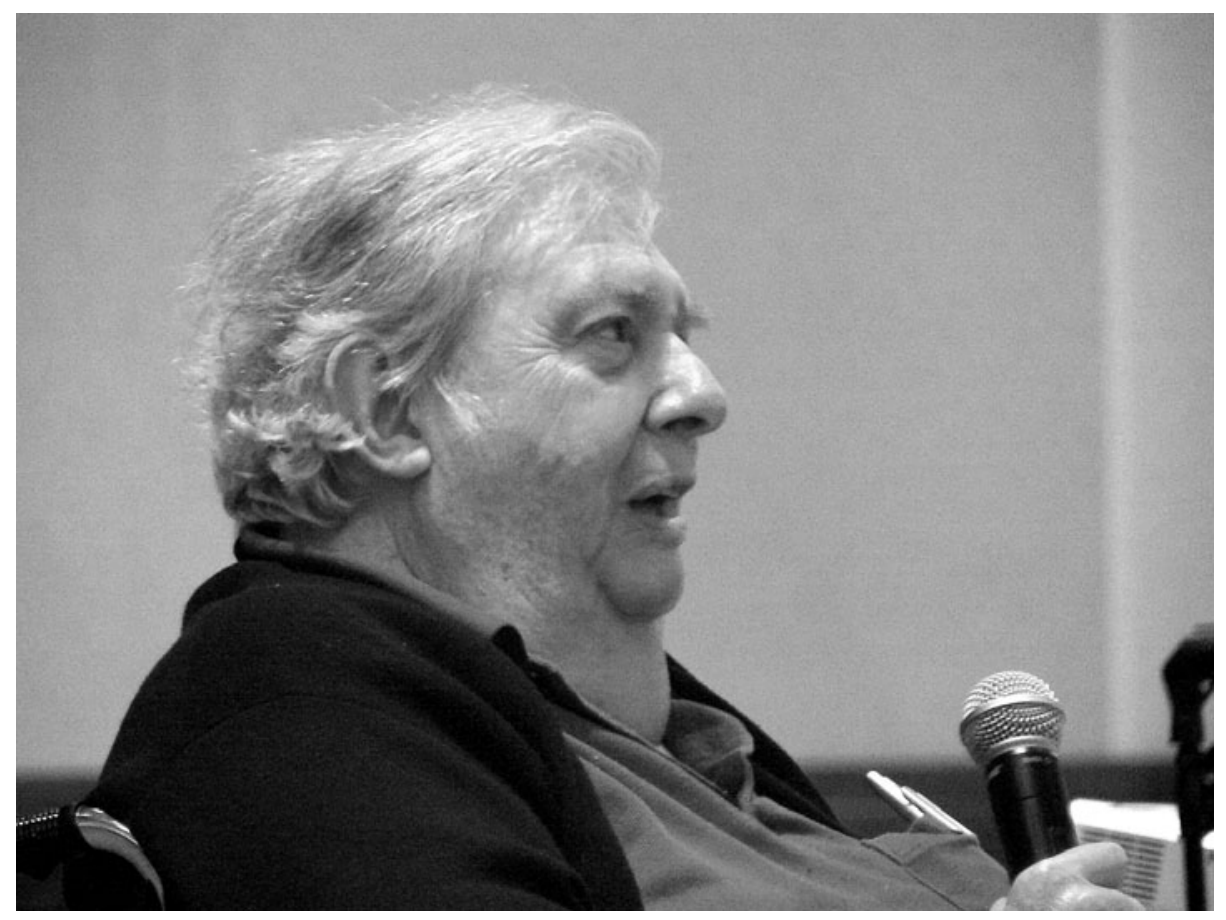

Geoffrey Burbidge. 